\title{
Exploring the Parsing of Dynamic Action in Checking Proneness
}

\author{
Sanaâ Belayachi ${ }^{1,2}$ and Martial Van der Linden ${ }^{1,2}$ \\ ${ }^{\prime}$ Cognitive Psychopathology and Neuropsychology Unit, University of Geneva, Geneva, Switzerland \\ ${ }^{2}$ Cognitive Neuroscience and Cognitive Psychopathology Unit, University of Liège, Liège, Wallonia, \\ Belgium
}

\begin{abstract}
Impaired action processing may be a key feature of the obsessive-compulsive checking phenomenon, although the mechanism underlying the impairment remains to be explored. We examined the ability to parse a continuous flow of movements and perceptual changes into meaningful segments of action - a key component of action processing - in checking proneness. Participants $(N=65)$ completed a measure of obsessive-compulsive symptoms and, while viewing four videotaped movies, were requested to detect the transitions between significant action steps. The main result indicated that Checking - but not the other obsessive-compulsive dimensions - was negatively related, with the size of meaningful units identified. These findings suggest that checking proneness may be specifically connected with difficulties in processing actions on the basis of abstract features such as goal-related information. This could explain why people with checking symptoms find it more difficult to determine whether an intended goal has actually been achieved.
\end{abstract}

Keywords: obsessive-compulsive disorder, checking, action processing, action parsing, event segmentation theory

In everyday situations, anyone may experience doubts about whether an intended action has actually been executed and, in response, may check for goal completion. According to some authors, this kind of difficulty in keeping track of one's actions (i.e., failing to determine whether an intended goal has been achieved) may occur when one fails to process an ongoing action's goal-related features (Vallacher \& Wegner, 1987). Certain contextual circumstances may trigger such a failure; in addition, some people may have a chronic failure in processing actions' goal features (Vallacher \& Wegner, 1989). This characteristic has been connected with an increased propensity to check everyday actions, similar to that observed in obsessive-compulsive disorder (OCD; Belayachi \& Van der Linden, 2009). Consistently, it has been suggested that people with OCD may lose track of the meaning of their behaviour because they process actions mainly according 'to low-level gestural units of behavior rather than to goal-related higher-level units' (Boyer \& Liénard, 2006, p. 1). People usually parse the continuous stream of behaviour into distinct meaningful action units (according to goal and subgoal completion). This allows one to understand what one is doing or what others are doing, with few resources allocated to the processing of each action detail. However, an inability to organise action details based on high-level meaningful features may impair the subjective understanding of what one has just done. This could in turn lead some people to deploy chronic checking behaviours, even at a subclinical

Address for correspondence: Sanâ̂ Belayachi, Cognitive Psychopathology and Neuropsychology Unit, University of Geneva, Geneva, Switzerland. E-mail: Sanaa.Belayachi@unige.ch

Behaviour Change | Volume 32 | Number 2| 2015 | pp. 93-103 | (C) The Author(s) 2015

doi $10.1017 /$ bec.2015.2 
level. This article aims to examine this assumption, by exploring whether checking proneness in the general population is characterised by a tendency to parse dynamic actions in terms of low-level gestural units.

Action parsing can be understood in the context of event segmentation theory (EST; Kurby \& Zacks, 2008; Zacks \& Sargent, 2010; Zacks \& Swallow, 2007; Zacks $\&$ Tversky, 2001). According to EST, online action processing combines bottomup chunking of perceptual information into basic action segments with top-down integration of these segments into larger action sequences. The latter process implies the ability to organise the numerous pieces of information gathered during action perception on the basis of high-level meaningful features, such as intentions, goals and subgoals. This naturally occurring kind of perceptual processing allows one to organise the abundant information of an action context (movement, perceptual changes) into chunks of meaningful information. In laboratory settings, it can be examined by asking participants to watch a movie and to press a button whenever they feel that one meaningful event is ending (e.g., subgoal attainment) and another one beginning. Numerous studies show that event-boundary judgments appear reliable within participants over time (Newtson, 1973; Zacks, Speer, Vettel, \& Jacoby, 2006).

The subjective perception of the boundaries separating action subparts depends on a 'perceiving by predicting mechanism', which allows the continuous flow of information to be processed with few resources. On the basis of event models, which represent invariants of a particular situation, people may make predictions about what is about to happen. Event models remain stable as long as the predictions based on them are correct; however, they are refined or replaced when sensory data contradict those predictions. This error-based updating mechanism is a key determinant of how a perceived action is segmented. Indeed, it has been suggested that error signals surrounding event model updating may mark event boundaries (Zacks, Kurby, Eisenberg, \& Haroutunian, 2011; Zacks \& Swallow, 2007). By using the video segmentation paradigm, it has been demonstrated that the segmentation rate increases as the action sequence becomes less predictable (e.g., Newtson, 1973). In other words, a lack of action parsing regarding goal state knowledge, as might be the case in checking, may result in a higher segmentation rate and in parsing the action into smaller parts (i.e., action parsing into low-level gestural units). Although event segmentation is a subjective task, numerous studies show that event-boundary judgments appear reliable across participants within the same sample (Newtson, 1973; Zacks et al., 2006). Indeed, by using the video segmentation paradigm, it has been demonstrated that most people identify inter-event boundaries around the same time points and that these normative boundaries generally refer to conceptual changes (e.g., changes in an actor's goal). Knowing that it is possible to calculate how close an individual's segmentation pattern is to the pattern for the entire sample (i.e., segmentation agreement), we assumed that a diminished ability to process actions' goal features would lead to lower segmentation agreement.

The main aim of the current study was to explore action parsing in checking proneness in light of the EST. Considering that a chronic focus on low-level gestural units of actions appears to be specific to checking (Belayachi \& Van der Linden, 2009), we expected checking proneness to be characterised by a tendency to identify smaller units of activity in a segmentation task. And knowing that low-level action parsing implies difficulties in processing actions' goal-related features, we also expected checking proneness to be connected with a decreased ability to identify inter-event boundaries in a normative way. We also examined the relationship between action 
parsing and memory in checking. Indeed, how actions are segmented may strongly influence the way people encode these actions in memory. For example, it has been shown that a reduced ability to identify significant event boundaries (i.e., poor segmentation agreement) may account for impaired performance on subsequent memory tasks (Kurby \& Zacks, 2011; Zacks et al., 2006). Given that checking proneness in non-clinical samples has been related to impaired memory for actions (e.g., Zermatten, Van der Linden, Larøi, \& Ceschi, 2006), we expected checking to be connected with poor memory performance; we also hypothesised that defective action parsing accounts for this relationship.

\section{Method}

\section{Participants}

Sixty-seven undergraduates who were randomly recruited from various faculties and schools at the University of Liège, all aged between 18 and 29, participated in the study. They were not compensated for their participation. Data from two participants had to be discarded as they appeared to be critical outliers (cf. Section 3). The reported results are from the remaining 65 participants ( 29 males and 36 females). Their mean age was 22 years $(S D=2.70$ years $)$.

\section{Apparatus: Event Segmentation Paradigm}

Event segmentation task. The segmentation task ${ }^{1}$ consisted of viewing four movies, each of which depicted an actor performing a familiar activity (setting up a tent, planting flowers, washing a car, and washing clothes). The mean duration of the movies was $378 \mathrm{~s}$. A fifth movie depicting the construction of a boat from toy blocks (160 s) was used for training. During movie viewing, participants were asked to identify units that were natural and meaningful to them by pressing the space bar whenever they felt that one event was ending and another one beginning. They were also told that there were no right or wrong answers in this task. Each participant started the segmentation task with the practice movie and then segmented all four movies (presented in random order). We computed the unit length, which refers to the mean duration of subevents identified. For each movie, we first divided the length of the movie by the number of boundaries identified. We then computed the mean unit length across the four movies. We also computed an index of how close a given participant's segmentation pattern was to the pattern for the entire sample (segmentation agreement). Movies were divided into 1-s bins. For each movie, we computed the proportion of participants who identified a boundary within each bin. Then, for each participant, we computed the point-biserial correlation between participant's segmentation data and the segmentation probabilities for the group.

Event-related memory tasks. Participants also completed recognition and order memory tests concerning the videos they had segmented (see Endnote 1). The recognition memory task consisted of pairs of pictures: one old picture taken from the segmented movie and one new picture taken from a visually similar movie that the participant had not seen. In each trial, an old picture and a new picture were selected at random and presented side by side on the screen, with the location of the old picture varying randomly. For each movie, 25 old pictures and 25 new pictures were presented to participants, with the instruction to identify the picture that had appeared in the movie they saw by pressing one of two buttons. We computed the proportion of correct 
responses (accuracy) and mean response latencies (reaction time). The order memory task consisted of 12 pictures (per movie), printed on $15.2-\mathrm{cm}$ by $10.2 \mathrm{-cm}$ paper with a white border, selected from the 25 pictures used in the recognition memory test. For each test, the pictures were placed in front of the participant in a pseudo-random order. Participants had to sort the pictures into the order in which they had occurred in the movie. We computed a completion time measure (time) and, on the basis of participants' sorting of the pictures, we calculated a measure of order error (deviation index), by recording the position in which each person placed each picture, determining the absolute deviation of that position from the correct position and averaging these deviations across each movie (lower error scores indicate better performance).

Picture Arrangement subtest. The Picture Arrangement subtest of the Wechsler Adult Intelligence Scale - III (Wechsler, 1997) was administered to ensure that our results did not depend on impaired semantic organisation and temporal sequencing in checking-prone people. This test requires participants to sort line drawings of everyday activities into the temporal order in which they typically occur in that activity.

\section{Self-Report Measures}

The Obsessive-Compulsive Inventory - Revised (OCI-R). The OCI-R (Foa et al., 2002) is an 18-item questionnaire evaluating six OC dimensions (checking, washing, obsessing, ordering, hoarding, and neutralising). The French version of the OCI-R (Zermatten, Van der Linden, Jermann, \& Ceschi, 2006) has good overall psychometric properties and a factor structure identical to that observed in Foa et al.'s (2002) original English version. In this study, Cronbach's alphas suggested adequate internal consistency for most subscales (with modest reliability for the neutralising scale; see Table 1). A statistical test of homogeneity confirmed that the mean OCI-R checking score of participants whose checking scores fell within the top quartile of the distribution $(N=15 ; M=5.9 ; S D=1.3)$ was comparable to that reported by Huppert et al. (2007) for $186 \mathrm{OCD}$ patients $(M=4.4 ; S D=3.6 ; p=.111)$.

The State-Trait Anxiety Inventory (STAI). The STAI (Spielberger, Gorsuch, \& Lushene, 1983) was administrated in order to control for the impact of anxiety on the level of action parsing, as high levels of anxiety can lead to local perceptual information processing (Derryberry \& Reed, 1998). The French version of the STAI (BruchonSchweitzer \& Paulhan, 1993) comprises 20 items assessing the respondents' state of anxiety at the time of the testing session (i.e., STAI-S) and 20 items constituting a self-reported measure of general anxiety (i.e., STAI-T). Both the STAI-S and the STAI-T show strong internal consistency in this study (see Table 1).

\section{Procedure}

Informed consent was obtained from all participants following an explanation of

96 the experimental procedure. In individual testing sessions, participants performed the segmentation task and the two memory tests, and completed the Picture Arrangement subtest and questionnaires assessing OCD and anxiety (as well as other questionnaires not presented in this article). The order of the questionnaires was counterbalanced across participants. Half of the participants first did the segmentation task, followed by the memory tests and then the Picture Arrangement subtest, after which they completed the questionnaires; whereas the other half completed the questionnaires first and then were given the remaining tasks. 


\section{TABLE 1}

Mean Scores and Standard Deviations $(S D)$ for the Segmentation Task, Two Memory Tests, $\mathrm{OCl}-\mathrm{R}$ and Anxiety Assessments, and Reliability for the Latter Two Sets of Variables

\begin{tabular}{lcc}
\hline Measures of interest & $\alpha$ & Mean (SD) \\
\hline OCl-R & .62 & $2.60(2.21)$ \\
Checking & .75 & $1.81(2.27)$ \\
Washing & .69 & $4.14(2.63)$ \\
Ordering & .79 & $3.80(2.73)$ \\
Hoarding & .73 & $2.58(2.36)$ \\
Obsessing & .57 & $1.14(1.63)$ \\
Neutralising & & \\
Anxiety & .87 & $31.31(6.83)$ \\
State anxiety & .89 & $41.94(8.69)$ \\
Trait anxiety & & $34.02(31.71)$ \\
Segmentation task & - & $0.31(1.30)$ \\
$\quad$ Unit length & - & \\
Segmentation agreement & & $0.71(1.24)$ \\
Recognition memory task & - & $3672(1113)$ \\
Accuracy & - & $0.93(.55)$ \\
Reaction time & & $98.64(34.16)$ \\
Temporal order memory task & - & $15.65(3.36)$ \\
$\quad$ Deviation index & - &
\end{tabular}

Note: Mean and $S D$ for the unit length, the proportion of correct responses in the recognition task and the six $\mathrm{OCl}-\mathrm{R}$ subscales are based on the nontransformed data.

\section{Results}

Non-normally distributed variables (the six OCI-R subscales, the unit length measure of the segmentation task and the accuracy variable of the recognition task) were transformed using the Box-Cox transformation to comply with normality and homogeneity of variance. Based on Cook's distance index, preliminary analyses identified two participants as multivariate outliers (subjects with a Cook's distance greater than the cutoff of 4 [number of cases - number of predictors - 1] are generally defined as multivariate outliers; Fox, 1991). We decided to exclude these data. Table 1 summarises the mean scores and standard deviation for the segmentation task, memory tests, Picture Arrangement subtest and OCI-R and anxiety measures.

\section{Relationships Between Checking and Action Parsing Measures}

Unit length. As Table 2 shows, there was a negative correlation between checking and unit length, which met the Bonferroni criterion for significance $(p<0.002)$. The obsessing and neutralising symptoms were also negatively correlated with unit length, at an uncorrected significance. 


\section{TABLE 2}

Pearson Correlations between OCl-R Subscales and the Segmentation Task Measures

\begin{tabular}{lcc}
\hline & \multicolumn{2}{c}{ Event segmentation } \\
\cline { 2 - 3 } OCI-R & Unit length & Segmentation agreement \\
\hline Checking & $-0.38^{*}(.002)$ & $.34(.006)$ \\
Washing & $-0.20(.102)$ & $-.02(.883)$ \\
Ordering & $-0.15(.244)$ & $.08(.555)$ \\
Hoarding & $0.10(.408)$ & $-.04(.780)$ \\
Obsessing & $-0.29(.020)$ & $.17(.178)$ \\
Neutralising & $-0.25(.045)$ & $.20(.105)$ \\
\hline
\end{tabular}

Note: Pearson correlations computed on the transformed unit length and OCl-R subscale measures, a Bonferroni correction of $(0.05 / 24)=0.002$ was used to correct for multiple comparisons, ${ }^{*}$ correlation significant at $p<0.002$.

Considering the potentially confounding influences of the intercorrelations between OCI-R subscales, zero-order correlations cannot determine the independent contribution of each $\mathrm{OC}$ dimension (i.e., once the effect of the other subscales has been removed). Hence, in order to investigate the specific relationship between the level of action parsing and the various OCI-R subscales, we performed a regression analysis with the transformed unit length measure as a dependent variable and the six transformed OCI-R subscales as independent variables, using the step-wise selection procedure. A Breusch-Pagan Test $\left(\chi^{2}=3.57, p=.735\right)$ suggests that the assumption of homoscedasticity is satisfied, and tolerance values (ranging from .54 to .83 ) and variance inflation factor (VIF) values (ranging from 1.19 to 1.87 ) suggest that there was no sign of multicollinearity (VIF values $>2.5$ and tolerance $<.40$ are considered as problematic; Allison, 1999). According to this regression analysis, checking is a significant independent predictor of low-level action parsing, $t=-3.17, p=.002$, $\beta=-.38$; whereas obsessing, $t=-1.56, p=.124, \beta=-.19$, and neutralising, $t$ $=-0.87, p=.390, \beta=-.12$, as well as washing, $t=-0.65, p=.520, \beta=-.08$, ordering, $t=-0.18, p=.858, \beta=-.02$, and hoarding, $t=1.66, p=.102, \beta=$ .19 , did not explain a significant proportion of variance when the other subscales are statistically controlled for.

We also examined the possibility that defective action parsing in checking could be secondary to deficits affecting organisational semantic strategies or to the fact that anxiety may increase attention to details. We therefore computed three separate partial correlations in order to re-examine the checking-unit length relation, once the Picture Arrangement subtest, state anxiety and trait anxiety scores were controlled for. These analyses revealed that the relationship between checking and unit length remained significant and was not explained by organisational semantic strategies, $r(62)=-.38, p=.002$, state anxiety, $r(62)=-.38, p=.002$, or trait anxiety, $r(62)$ $=-.36, p=.003$.

Segmentation agreement. The results reported in Table 2 revealed a positive correlation between checking and segmentation agreement that did not meet the Bonferroni criterion for significance. 


\section{TABLE 3}

Pearson Correlations Between Memory Performance and Checking and the Segmentation Task Measures

\begin{tabular}{lrcr}
\hline & \multicolumn{2}{c}{ Event segmentation } & \\
\cline { 2 - 3 } & Unit length & Segmentation agreement & $\begin{array}{l}\text { OCl-R } \\
\text { Checking }\end{array}$ \\
\hline Recognition memory & & & \\
$\quad$ Accuracy & $-.02(.863)$ & $-.12(.361)$ & $-.02(.902)$ \\
Reaction time & $-.05(.685)$ & $.14(.268)$ & $.17(.165)$ \\
Temporal order & & & $-.02(.880)$ \\
$\quad$ Deviation index & $.05(.680)$ & $-.13(.306)$ & $.01(.913)$ \\
Time & $-.07(.587)$ & $.16(.203)$ & \\
\hline
\end{tabular}

Note: Pearson correlations computed on the transformed unit length, accuracy and checking measures.

\section{Checking, Action Parsing and Memory Performance}

A secondary aim of this study was to examine whether defective segmentation may contribute to memory deficits in checking. As Table 3 reveals, memory performance was not related to action parsing measures or to checking proneness.

\section{Discussion}

The main aim of the present study was to explore the parsing of dynamic action in checking proneness, by using a task that requires participants to detect meaningful action units while viewing videotaped familiar actions. As expected, we observed a negative correlation between checking and the length of the action units identified, which is not secondary to a general deficit affecting semantic organisational capacities or to anxiety. Contrary to our hypothesis, checking was connected with good segmentation agreement, suggesting that this OC symptom may not be connected with problems identifying significant boundaries during action parsing. Nevertheless, we cannot exclude the possibility that this relation may result from the tendency to parse at a low level, thus increasing the probability that one will identify inter-event boundaries at the same time points as the entire sample.

Overall, these results suggest that checking-prone people may rely predominantly on low-level movement cues to understand actions. To our knowledge, this is the first empirical support for the theoretical assumption that some repetitive and/or ritualised behaviours might be characterised by a type of action parsing that predominantly considers low-level gestural action units (e.g., fine motor changes), rather than abstract meaningful features (e.g., goal changes; Boyer \& Liénard, 2006; Zacks \& Sargent, 2010). Our results are also in agreement with previous studies of action processing in OCD. Indeed, people with OCD were found to have an impaired ability to understand biological motions in terms of intentions and goals (see Jung et al., 2009; Kim et al., 2008). In another study, individuals with checking proneness appeared to construe various familiar actions mainly in terms of their procedural aspects and motor components, rather than according to the related goals and abstract outcomes (Belayachi \& Van der Linden, 2009). The phenomenology of checking has frequently been connected with an increased tendency to generate an error signal at the early 
stages of action evaluation (i.e., overactive action monitoring; e.g., Ursu, Stenger, Shear, Jones, \& Carter, 2003). This excessive error signalling in OCD is thought to originate in the anterior cingulate cortex (ACC), which was shown to be hyperactivated only among checking individuals (Murayama et al., 2012). Interestingly, EST states that the detection of inter-event boundaries may stem from error prediction signals generated when predictions about what is about to happen mismatch what is actually perceived; the ACC may be responsible for generating such signals (Kurby \& Zacks, 2008; Zacks \& Sargent, 2010; Zacks, Speer, Swallow, \& Maley, 2010). Knowing that a recent brain imaging study of event segmentation during text reading (Speer, Zacks, \& Reynolds, 2007) showed that the cingulate cortex, in addition to other brain areas, is particularly sensitive to the presence of an event boundary, thus, low-level action parsing in checking could stem from overactivity in the ACC. Future studies should be conducted to directly examine the association between the neurocognitive markers of the increased error signal and low-level action parsing in checking.

Meanwhile, the origin of low-level action parsing in checking must be further explored. According to EST, participants may use low-level movement cues to segment dynamic action when they fail to perceive it as goal-directed; however, they may depend less on these cues when they can rely on top-down knowledge based on previous experience with analogous situations (Zacks, 2004). In light of this framework, one could suggest that checking-prone people may be unable to use such abstract representations for goal processing, which may lead them to rely more on low-level gestural cues in order to understand what they are doing or have just done. It must also be determined whether this way of functioning reflects a spontaneous focus on low-level gestural parsing or constitutes a strategy to compensate for an impaired ability to use abstract representations for goal understanding. This issue could be examined by using different task instructions explicitly asking participants to segment the perceived action at a coarse grain.

A secondary aim of this study was to examine whether defective action parsing might account for impaired memory in checking. Unexpectedly, checking was not related to poor memory performance. Action parsing measures were not connected with memory performance either. It should be noted that a connection between segmentation measures and memory performance has been observed only among elderly participants (Kurby \& Zacks, 2011; Zacks et al., 2006) and participants diagnosed with mild Alzheimer disease (AD; Zacks et al., 2006, experiment 2). More specifically, both elderly and AD participants were characterised by a decreased ability to identify the significant inter-event boundaries identified by the entire sample (i.e., poor segmentation agreement). Among elderly and AD participants, low segmentation agreement was connected with impaired performance on both the temporal ordering and recognition memory tasks, whereas no such association was observed among young participants. Contrary to this finding, our checking participants were not characterised by an impaired ability to identify significant inter-event boundaries (i.e., good segmentation agreement), which may explain why we did not observe poor memory performance in checking. As well, the nature of memory deficits in checking is different from those in AD. Checking people may have problems with active recollection, which may be secondary to executive dysfunctions (Savage et al., 2000). Indeed, people with OCD are more prone to focus on details and less able to transfer their attention to larger components of a scene, which may in turn lead to fragmented encoding (for a review, see Harkin \& Kessler, 2011). In this context, the relationship 
between action parsing and memory might be better understood by using a free recall task; for example, by asking participants to recall the main steps of the segmented activities.

Before concluding, we will briefly address some limitations of this study. First, our results do not allow us to determine the nature of the relationship between action parsing and checking proneness; whether chronically low-level gestural parsing leads people to check their actions, or whether frequent checking behaviour may, in the long run, provoke aberrant action processing remains to be explored. Second, although checking in non-clinical samples have been found to have clinical features and cognitive impairments similar to those identified in OCD patients in a wide range of studies (for a critical review, see Muller \& Roberts, 2005), further studies should be conducted to replicate our findings in individuals with more severe checking symptoms (i.e., OCD patients).

\section{Conclusions}

The results of this study suggest that an impaired ability to process actions in terms of goal-related features could, in association with other factors, lead some people to deploy checking behaviours. The mechanism underlying low-level action parsing in checking remains to be further explored. Future studies should also be conducted to examine whether checking people are able to parse at a coarse grain, when they are instructed to do so. Finally, future research is needed to replicate these findings in a clinical sample.

\section{Acknowledgments}

This study was supported by a grant from the French-speaking community of Belgium (Action Recherche Concertée, Convention 06/11-340).

\section{Endnote}

${ }^{1}$ Materials for the segmentation task and the two memory tests were kindly provided by Professor Jeffrey Zacks. For a detailed description of all the stimuli, see Zacks et al. (2006; experiment 2).

\section{References}

Allison, P.D. (1999). Logistic regression using the SAS system: Theory and application. Cary, NC: SAS Institute.

Belayachi, S., \& Van der Linden, M. (2009). Level of agency in sub-clinical checking. Consciousness and Cognition, 18, 293-299.

Boyer, P., \& Liénard, P. (2006). Precaution systems and ritualized behavior. Behavioral and Brain Sciences, 29, 635-641.

Bruchon-Schweitzer, M., \& Paulhan, I. (1993). Le manuel du STAI-Y de CD Spielberger, adaptation française. Paris: ECPA.

Derryberry, D., \& Reed, M.A. (1998). Anxiety and attentional focusing: Trait, state and hemispheric influences. Personality and Individual Differences, 25, 745-761.

Foa, E.B., Huppert, J.D., Leiberg, S., Langner, R., Kichic, R., Hajcak, G., \& Salkovskis, P.M. (2002). The Obsessive-Compulsive Inventory: Development and validation of a short version. Psychological Assessment, 14, 485-496.

Fox, J. (1991). Regression diagnostics: An introduction. Newbury Park, CA: SAGE Publications. 
Harkin, B., \& Kessler, K. (2011). The role of working memory in compulsive checking and OCD: A systematic classification of 58 experimental findings. Clinical Psychology Review, 31, 10041021.

Huppert, J.D., Walther, M.R., Hajcak, G., Yadin, E., Foa, E.B., Simpson, H.B., \& Liebowitz, M.R. (2007). The OCI-R: Validation of the subscales in a clinical sample. Journal of Anxiety Disorders, 21,394-406.

Jung, W.H., Gu, B.M., Kang, D.H., Park, J.Y., Yoo, S.Y., Choi, C.H., .. Kwon, J.S. (2009). BOLD response during visual perception of biological motion in obsessive-compulsive disorder. European Archives of Psychiatry and Clinical Neuroscience, 259, 46-54.

Kim, J., Blake, R., Park, S., Shin, Y.W., Kang, D.H., \& Kwon, J.S. (2008). Selective impairment in visual perception of biological motion in obsessive-compulsive disorder. Depression and Anxiety, 25, e15-e25.

Kurby, C.A., \& Zacks, J.M. (2008). Segmentation in the perception and memory of events. Trends in Cognitive Sciences, 12, 72-79.

Kurby, C.A., \& Zacks, J.M. (2011). Age differences in the perception of hierarchical structure in events. Memory $\mathcal{E}$ Cognition, 39, 75-91.

Muller, J., \& Roberts, J.E. (2005). Memory and attention in obsessive-compulsive disorder: A review. Journal of Anxiety Disorders, 19, 1-28.

Murayama, K., Nakao, T., Sanematsu, H., Okada, K., Yoshiura, T., Tomita, M., ... Kanba, S. (2012). Differential neural network of checking versus washing symptoms in obsessive-compulsive disorder. Progress in Neuro-Psychopharmacology and Biological Psychiatry, 40, 160-166.

Newtson, D. (1973). Attribution and the unit of perception of ongoing behavior. Journal of Personality and Social Psychology, 28, 28-38.

Savage, C.R., Deckersbach, T., Wilhelm, S., Rauch, S.L., Baer, L., Reid, T., \& Jenike, M.A. (2000). Strategic processing and episodic memory impairment in obsessive compulsive disorder. Neuropsychology, 14, 141-151.

Speer, N.K., Zacks, J.M., \& Reynolds, J.R. (2007). Human brain activity time-locked to narrative event boundaries. Psychological Science, 18, 449-455.

Spielberger, C.D., Gorsuch, R.L., \& Lushene, R.E. (1983). Manual for the state-trait anxiety inventory (Form Y). Palo Alto, CA: Consulting Psychologists Press.

Ursu, S., Stenger, V.A., Shear, M.K., Jones, M.R., \& Carter, C.S. (2003). Overactive action monitoring in obsessive-compulsive disorder: Evidence from functional magnetic resonance imaging. Psychological Science, 14, 347-353.

Vallacher, R.R., \& Wegner, D.M. (1987). What do people think they're doing? Action identification and human behavior. Psychological Review, 94, 3-15.

Vallacher, R.R., \& Wegner, D.M. (1989). Levels of personal agency: Individual variation in action identification. Journal of Personality and Social Psychology, 57, 660-671.

Wechsler, D. (1997). Wechsler Adult Intelligence Scale - III. San Antonio, TX: Psychological Corporation.

Zacks, J.M. (2004). Using movement and intentions to understand simple events. Cognitive Science, 28, 979-1008.

Zacks, J.M., Kurby, C.A., Eisenberg, M.L., \& Haroutunian, N. (2011). Prediction error associated with the perceptual segmentation of naturalistic events. Journal of Cognitive Neuroscience, 23, $4057-4066$.

Zacks, J.M., \& Sargent, J.Q. (2010). Event perception: A theory and its application to clinical neuroscience. Psychology of Learning and Motivation, 53, 253-299.

Zacks, J.M., Speer, N.K., Swallow, K.M., \& Maley, C.J. (2010). The brain's cutting-room floor: Segmentation of narrative cinema. Frontiers in Human Neuroscience, 4, 1-14.

Zacks, J.M., Speer, N.K., Vettel, J.M., \& Jacoby, L.L. (2006). Event understanding and memory in healthy aging and dementia of the Alzheimer type. Psychology and Aging, 21, 466-482.

Zacks, J.M., \& Swallow, K.M. (2007). Event segmentation. Current Directions in Psychological Science, $16,80-84$. 
Zacks, J.M., \& Tversky, B. (2001). Event structure in perception and conception. Psychological Bulletin, 127, 3-21.

Zermatten, A., Van der Linden, M., Jermann, F., \& Ceschi, G. (2006). Validation of a French version of the Obsessive-Compulsive Inventory - Revised in a non-clinical sample. Revue Européenne de Psychologie Appliquee/European Review of Applied Psychology, 56, 151-155. 